\title{
Design of PLC Control System for Automatic Door
}

\author{
Jiangshan Gao, Yan Zhi \\ College of Mechanical and Control Engineering, Guilin University of Technology, Guilin, China \\ Email: 56582650@qq.com
}

How to cite this paper: Gao, J.S. and Zhi, Y. (2019) Design of PLC Control System for Automatic Door. Open Access Library Journal, 6: e5891.

https://doi.org/10.4236/oalib.1105891

Received: October 30, 2019

Accepted: November 10, 2019

Published: November 13, 2019

Copyright ( 2019 by author(s) and Open Access Library Inc.

This work is licensed under the Creative Commons Attribution International License (CC BY 4.0).

http://creativecommons.org/licenses/by/4.0/

(c) (i) Open Access

\begin{abstract}
The core of automatic door is its control system. The basic principle of automatic door control system is to use PLC to output digital signal and analog signal to inverter to change working power frequency and drive motor steering, drive motor frequency conversion, change direction operation, drive automatic door to realize door opening and closing operations at different speeds. When the signal acquisition device of the automatic door detects whether a person or an object has passed, the automatic door can be automatically opened and closed to realize the function of the person coming to the door and the person to close the door. The system designed in this paper not only completes the opening and closing of the automatic door at different speeds under different conditions, but also adds more safety protection, such as anti-pinch control, emergency stop and system fault self-test, to achieve the expected design effect. The automatic door control system has the characteristics of higher safety, ease of use and stability.
\end{abstract}

\section{Subject Areas}

Mechanical Engineering

\section{Keywords}

Automatic Door, PLC, Signal Acquisition Device, Frequency

\section{Introduction}

The figure of the automatic door in life can be seen everywhere. The main working principle of the automatic door is to sense whether there is an object passing through the sensing device at the top of the door and the auxiliary infrared sensing device on the side of the door frame, giving the door a switch signal and then programmable control. The device, the frequency converter, the drive 
motor and the transmission control the movement of the door leaf to realize the function of opening and closing the door.

The biggest feature of the automatic door is that it can complete the automatic door opening and closing action without being supervised, which gives people great convenience, but the biggest problem in the case of no one is the safety hazard. The popularity of automatic doors in China is not wide enough, which leads to the slow development of automatic doors. The system construction relies mainly on imports. The control part of the hardware cannot meet the market demand. The software design is relatively simple, and the designed system is relatively simple [1]. These can determine the automatic door, the stability and safety of operation, so China needs to strengthen the research of control system, combined with the foreign automatic door function and local actual needs, using advanced software compilation, solving various problems existing in the development process of automatic door, and promoting the automatic door industry.

The design of this paper is an automatic door applied to public places such as shopping malls and high-end hotel entrances and exits, which require strong security and practicality. Integrating automatic door scenes and usage habits in daily life, this paper designs the most used automatic sliding door [2]. The automatic sliding door uses the inductive switch and safety grating to realize automatic control. At the same time, the manual control is realized by using the operation buttons, and the use is made. Auxiliary devices such as backup power supply to meet the needs of different situations of adult traffic.

\section{System Design}

The design of the automatic door needs to be considered for the purpose of safety and simplicity. This design is applied to public places with large traffic flow. It is designed to take care of the practicality of practical use and improve convenience [3]. The design of automatic door control system based on PLC needs to consider the practicality and safety in real life, and ensure the safety of the passing object under the guarantee of smooth operation of the automatic door. This paper adopts the photoelectric sensor to collect whether the object passes the signal to the PLC, and controls the opening and closing of the automatic door through the inverter, the driving motor and the transmission device in the PLC control actuator; meanwhile, the infrared sensor is opened after the automatic door is opened. Let the relevant implementing agencies coordinate work to ensure that the automatic door can operate normally and safely, as shown in Figure 1.

The main content of the automatic door control system design process includes the following aspects: master the control principle and control flow of the automatic door, and understand the control process of the automatic door as a whole. The input signal device and the output control device are clearly designed, and the number of input and output points is estimated in combination with the requirements of the automatic door function design. Select the appropriate PLC type based on the number of I/O points. According to the number of 


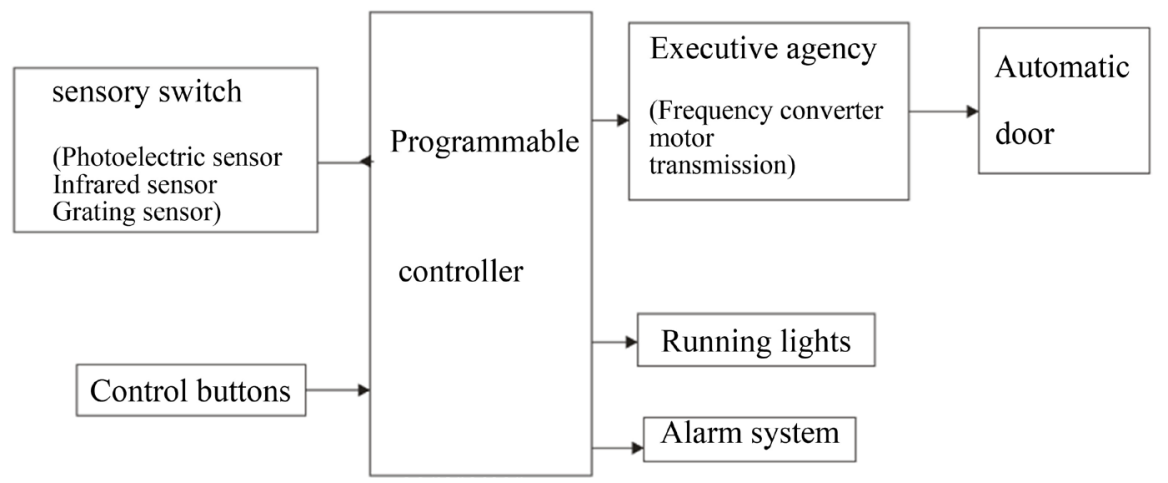

Figure 1. Schematic diagram of the automatic door control system.

I/O points, the input and output addresses are reasonably distributed, and the terminal wiring diagram is drawn, which is beneficial to the design of the ladder diagram and the convenience in using the simulation. Draw a complete ladder diagram according to the curriculum design requirements and practical applications. Use software simulation to export the program to the specified format and run the simulation to see if the function is implemented [4]. Troubleshoot and unreasonable troubleshooting procedures, optimize program design; optimize overall debugging of automatic door systems, optimize problematic programs, and continuously improve the system through repeated simulations until the desired functions are implemented.

\section{System Hardware Design}

In this paper, the drive motor drives the transmission device to realize the opening and closing of the automatic door. The operation of the motor is realized by the AC frequency controlled by the PLC to control the frequency converter to supply the motor. The advantages of this design are easy operation, stable operation and high real-time performance. Compared with other types of motors, more mature AC motors can control cost, have stable torque output and a larger speed range. Improve the stability of the long-term operation of the automatic door, the system hardware components, as shown in Figure 2.

The transmission of the automatic door is mainly composed of a drive motor, a transmission belt and a transmission pulley. Considering the convenient installation and maintenance, the transmission only occupies a small part of the whole system. For example, the pulley that drives the door body needs to be fixed outside the wall to be detachable structure. The three-phase asynchronous motor is selected as the drive motor. Passing three-phase symmetrical alternating current into the stator winding of the motor generates a circular rotating magnetic field. In the magnetic field, the rotor winding cuts the magnetic field to generate an induced current, and the rotor winding in the closed path also generates an electromagnetic force. Under the interaction of the two magnetic fields, the motor, the electromagnetic torque in the same direction as the rotating magnetic field is formed on the rotating shaft, so that the rotor of the motor rotates [5]. 


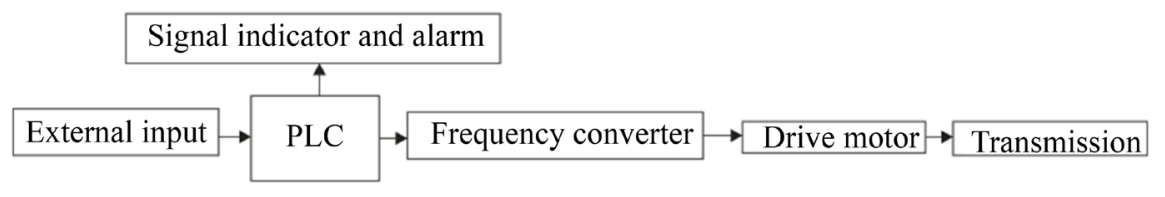

Figure 2. Hardware structure of the system.

For the selection of automatic door drive motor, YS series motor is selected. The fully enclosed YS series three-phase asynchronous motor uses E-class motor protection. ICO141 is used for cooling method. It has light weight, low noise, stable operation and structure, simple and economical and energy efficient. Based on the stability and smoothness required in the design of this paper and the consideration of economical and practical aspects, the YS series motor was selected, and the model used was YS6314.

The automatic door designed in this paper is used in a public place with a large flow of people, so it is necessary to select a sensitive device with high sensitivity. In this paper, the sensor switch mainly uses infrared human body sensor switch, microwave radar sensor switch and safety grating. Combined with actual needs, the following are the selection of each sensor [6]. When the automatic door is closed, when the safety grating detects that an object has passed, the door body stops descending, and the automatic door automatically moves up to play a safety guarantee function.

The I/O address allocation of the automatic door control system is written according to the number of input and output signals required by the automatic door control system, and are also the index data for writing the PLC program and performing system debugging. According to the estimation of the number of input and output addresses, the design selects the CPU 226 as the configured S7-200 PLC, and needs to input 12 points and 14 output points [7].

\section{System Software Design}

The basic functions realized by this design are as follows: 1) When there is no data connection with the PLC, the new file can be edited according to actual needs, the error location is checked, the program and system configuration are modified, and the program is stored in the hard disk of the PC. 2) Under the online communication, the software uploads and downloads data and programs, and can directly operate the PLC, such as editing programs and modifying instructions. When writing a program is an error, the software can point out some of the errors entered. When writing a ladder diagram, when there is a program problem, the dialog window below can directly indicate the problem of the network program that has the problem, and the user can modify the program according to the prompt. 3) At the menu bar of the software, the user can use the response function module according to the prompt. 4) The user can modify the PLC configuration, communication settings, and work type, and can also monitor the operation of the PLC. The system flow is shown in Figure 3.

Automatic control of closing and anti-clamping: The automatic door touches 


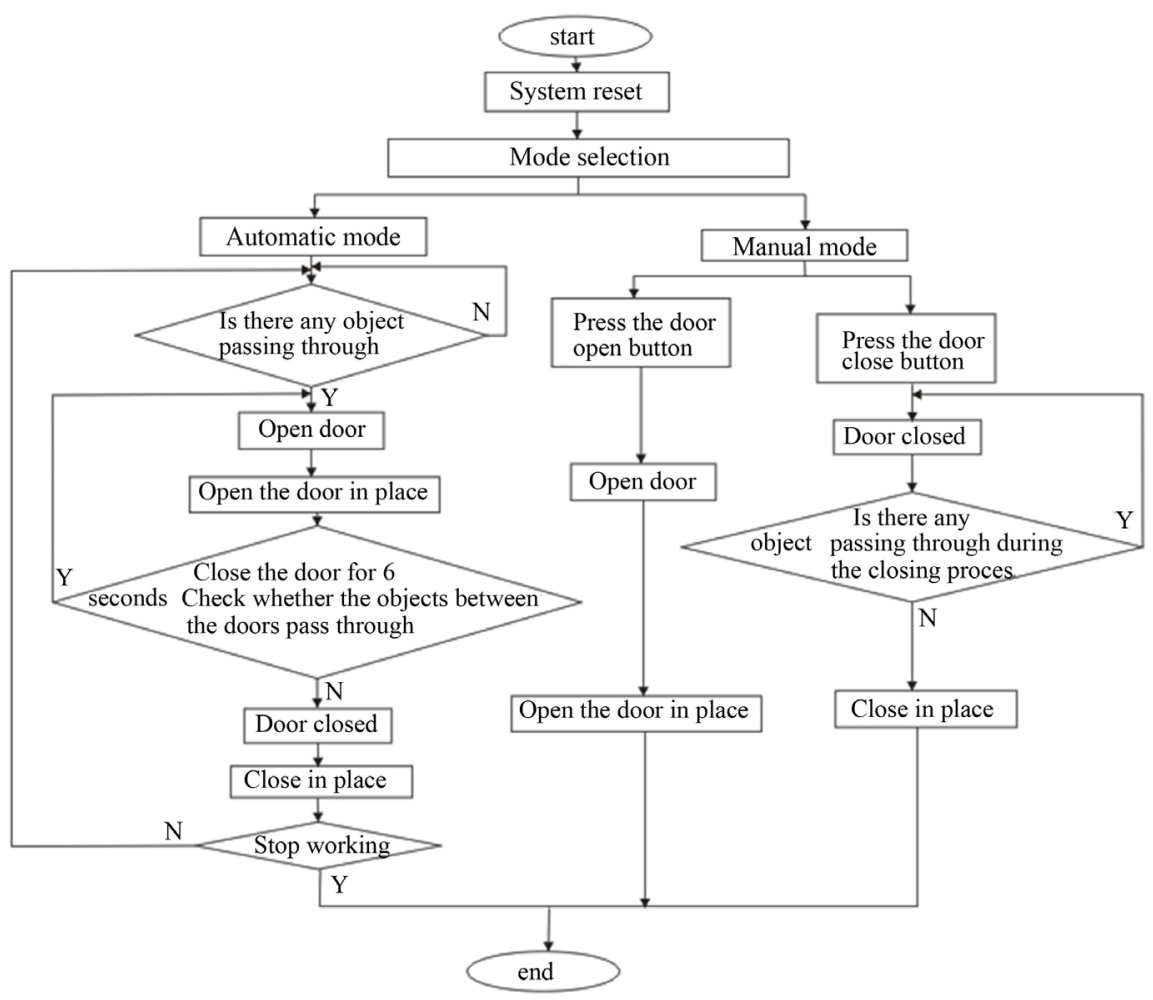

Figure 3. System flow chart.

the door opener I0.4 and enters the 8s timing. During the three sensors I0.6, I0.7, I1.0, if an object is detected, the timer T37 retimed. After the automatic door detects no object for $8 \mathrm{~s}$, the PLC's Q0.1, M0, V0 action, pass to the inverter a motor reversal signal and low speed running signal, a total of $6 \mathrm{~s}$ automatic door closing, if any object is closed when the automatic door is closed I1.0 detects that the automatic door stops closing and executes the automatic door opening procedure. In the automatic closing, due to the fact that the object passes the fast factor, the automatic door uses a safety grating with a delay of only $0.2 \mathrm{~s}$ to prevent the object from being clamped.

There are two kinds of alarm conditions in the automatic door. The first one is the automatic door program. The automatic door appears to be operated manually or automatically (Q0.0 or Q0.2) and closed (Q0.1 or Q0.3). The door will judge by the program, the M0.1 action will stop the whole automatic door control system, the fault light Q1.1 will light up, remind the staff to come to check, after checking no problem, press the abnormal reset button I1.3 to make the automatic The gate program is reset.

When a person or an object is not detected by the automatic door, in order to prevent the secondary control from being caused by the manual control, the surrounding personnel can directly press the red emergency stop button I0.1, and the M0.0 action automatic door program stops working, and then For rescue or treatment, the alarm light Q0.5 works simultaneously with the alarm Q0.4 to remind the problem. 


\section{System Verification}

When the automatic door encounters an emergency, and presses the emergency stop button I0.1, the automatic door stops all work, the alarm Q0.4 and the alarm light Q0.5 work at the same time. After the check is normal, it presses the abnormal reset button I1.3 automatically. The door is re-worked. The automatic door in the text is mainly composed of two control modes, manual control and automatic control of the opening and closing of the automatic door. When the automatic control is run, the actuator is opened and closed according to whether or not someone has changed the frequency of the inverter through the system.

\section{Conflicts of Interest}

The authors declare no conflicts of interest regarding the publication of this paper.

\section{References}

[1] Jiang, J.F. (2010) Siemens S7-200 PLC Engineering Application Technology Tutorial. Mechanical Industry Press, Beijing.

[2] Xiang, X.H. (2010) Siemens S7 PLC Advanced Application Examples Explained. Mechanical Industry Press, Beijing.

[3] Zhao, J.B. (2011) The Example Explains the Siemens S7-200PLC from Entry to the Essence. Publishing House of Electronics Industry, Beijing, 21-30.

[4] Gao, H. (2011) PLC Sequential Control Design Method and Its Application. Hengyang Finance and Industry Vocational and Technical College, No. 4, 145-146.

[5] Hu, Y.Y. (2017) PLC Automatic Door Working Principle. Suffolk University School of Science and Technology, No. 16, 247-248.

[6] Tao, J. (2017) Automatic Sliding Door Design Based on PLC. Science and Technology Information, No. 19, 55-58.

[7] Lei, L.H. (2019) Automatic Door System Based on PLC control. GEZE Industrial (Tianjin) Co., Ltd., Tianjin. 